\title{
RESPIRATORY TRACT INFECTION
}

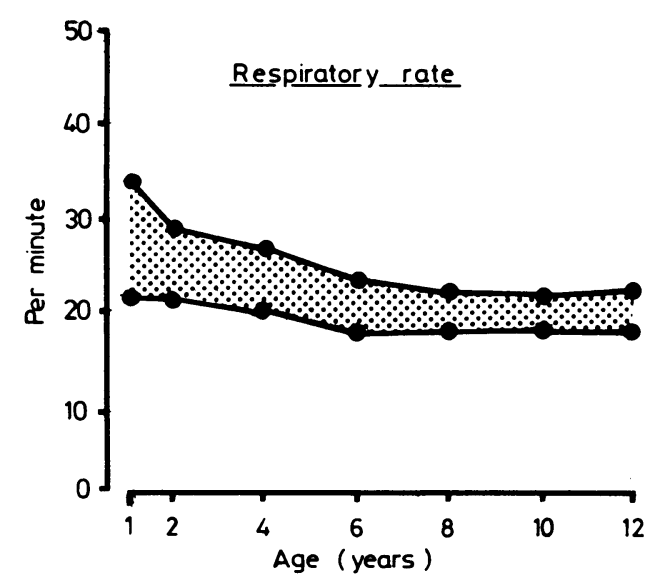

Normal range
Infection of the respiratory tract is a common cause of illness in children. Although pathogens are often not confined to anatomical boundaries, the infections may be classified as:

(a) upper respiratory tract-common cold, tonsillitis and pharyngitis, and acute otitis media;

(b) middle respiratory tract-acute laryngitis and epiglottitis;

(c) lower respiratory tract-bronchitis, bronchopneumonia, and segmental pneumonia.

Viruses, which cause most respiratory tract infections, and bacterial infections produce similar clinical illness. Different viruses may produce an identical picture, or the same virus may cause different clinical syndromes. Clinically it may not be possible to determine whether the infection is due to viruses, bacteria, or both. If the infection is suspected of being bacterial it is safest to prescribe an antibiotic, as the results of virus studies are often received after the acute symptoms have passed. The commonest bacterial pathogens are pneumococci. Less common are Haemophilus influenzae, group A $\beta$-haemolytic streptococci, Staphylococcus aureus, group B $\beta$-haemolytic streptococci, Gram-negative bacteria, and anaerobic bacteria.

Preschool children usually have about six colds each year. The main symptoms are sneezing, nasal discharge, and mild fever. Similar symptoms may occur in the early phases of infection with rotavirus and be followed by vomiting and diarrhoea. Postnasal discharge may produce coughing. The commonest complication is acute otitis media, but secondary bacterial infection of the lower respiratory tract sometimes occurs.

There is no specific treatment for the common cold, and antibiotics should not be given. A danger with nasal drops is that they will run down into the lower respiratory tract and carry the infection there.

Acute bronchitis often follows a viral upper respiratory tract infection and there is always a cough, which may be accompanied by wheezing. There is no fever. The respiratory rate is normal and the symptoms resolve within a week. The only signs, which are not constantly present, are rhonchi. Since it is usually due to a virus antibiotics are indicated only if the illness is severe or a bacterial cause is shown. 


\section{Recurrent bronchitis}

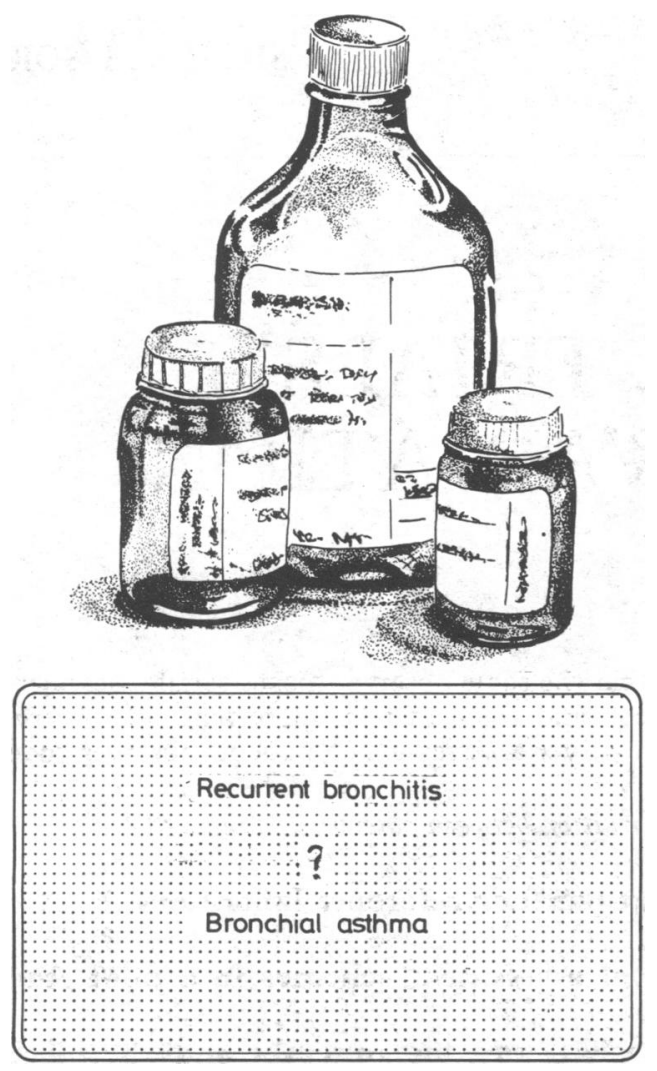

Although all concerned with children are familiar with the catarrhal child, the exact pathology of the condition is unknown, and it is called by many names-postnasal discharge, perennial rhinitis, or recurrent bronchitis. These children have an increased incidence of colds, tonsillitis, and acute otitis media. The symptom of recurrent cough without wheezing is most common during the second half of the first year of life, the first two years of nursery school, and the first two years at primary school. Between episodes of cough the child has at least one week when he is completely free of symptoms. Recurrent viral or bacterial infection contracted from siblings or fellow pupils may be important, but the considerable differences between the behaviour of children in the same family suggest the possibility of a temporary immunological defect.

Various treatments including nasal drops and oral preparations of antihistamines are given with little effect. A chest radiograph should be performed to exclude persistent segmental or lobar collapse. A Mantoux test for tuberculosis should be performed, a sweat test to exclude cystic fibrosis, and plasma immunoglobulin studies to exclude rare symptoms.

Some infants with a family history of hayfever, asthma, or eczema suffer recurrent episodes of cough and wheeze, especially in the winter. These episodes are often preceded by nasal discharge and sometimes accompanied by fever. The children are not severely ill and rhonchi are present. Antibiotics and bronchodilators have little effect. Symptoms gradually subside within a few days even without treatment. Most children lose all these symptoms by the age of 4 years, though a few then show the characteristic features of bronchial asthma. If a child becomes severely ill during these recurrent attacks the possibility of bronchial asthma should be considered by a paediatrician (see article on bronchial asthma).

\section{Bronchopneumonia and segmental pneumonia}
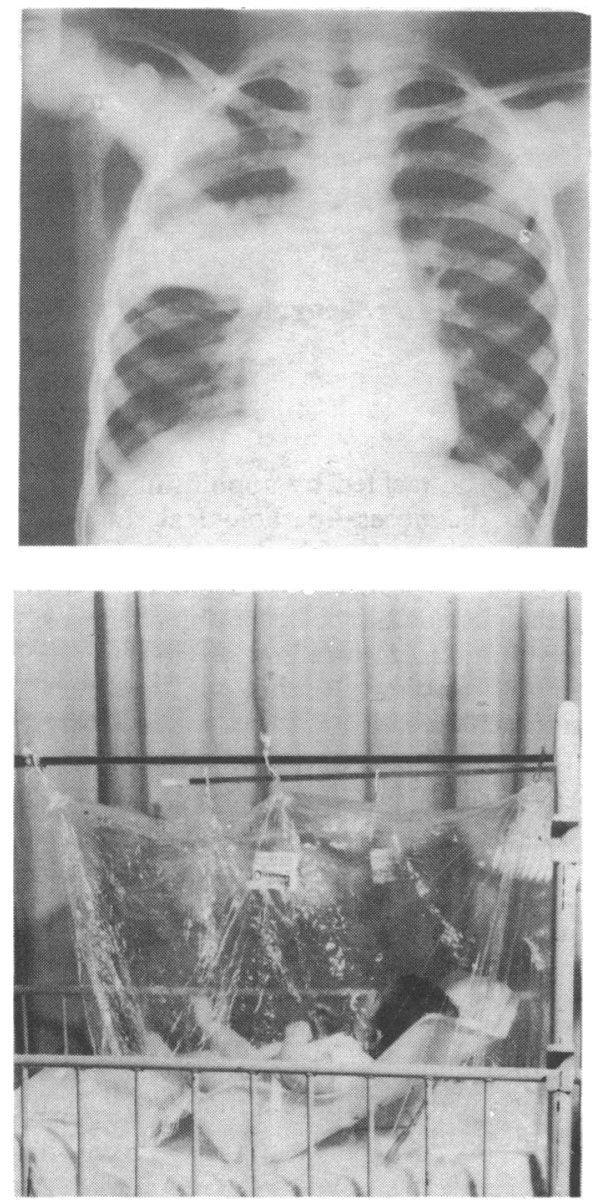

Pneumonia is acute inflammation of the lung alveoli. In bronchopneumonia the infection is spread throughout the bronchial tree whereas in segmental pneumonia it is confined to the alveoli in one segment or lobe. A raised respiratory rate at rest distinguishes pneumonia from bronchitis. Cough, fever, and flaring of the alae nasi are usually present and there may be reduced breath sounds over the affected area as well as crepitations. A chest radiograph, which is needed in every child with suspected pneumonia, may show extensive changes when there are no localising signs in the chest. The radiograph may show an opacity confined to a single segment or lobe but there may be bilateral patchy changes. Bacterial cultures of throat swabs and blood should be performed before treatment is started. Ideally virological studies of nasopharyngeal secretions and estimations of virus antibody titres in sera collected in the acute and convalescent phases should also be done.

Children with pneumonia are best treated in hospital as they often need oxygen treatment. Antibiotics should be prescribed for all cases of pneumonia, although later a viral cause may be discovered. Ampicillin and cloxacillin are given intravenously by bolus injection for bronchopneumonia, whereas penicillin or ampicillin can be used in segmental pneumonia as the pneumococcus is the commonest pathogen. Severely ill children may have either staphylococcal pneumonia or a Gram-negative infection and they need an additional antibiotic. Fusidic acid is added for suspected staphylococcal infection and gentamicin for Gram-negative infection. Oral erythromycin may be given to patients who are sensitive to penicillins and to those whose failure to respond to the other antibiotics suggests that they have mycoplasma infection. Antibiotic treatment can be modified when the results of bacterial cultures are available. Intravenous fluids may be needed and oxygen treatment is best provided in a tent. 